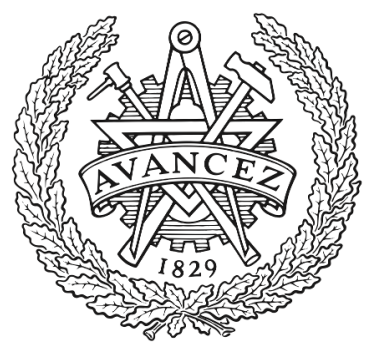

CHALMERS

UNIVERSITY OF TECHNOLOGY

\title{
A Multilayer-Stacked UWB Yagi Antenna
}

Downloaded from: https://research.chalmers.se, 2023-04-26 13:38 UTC

Citation for the original published paper (version of record):

Wan, K., Xie, C., Zheng, Y. et al (2017). A Multilayer-Stacked UWB Yagi Antenna. 2017

International Symposium on Antennas and Propagation, ISAP 2017, Volume 2017-January: 1-2.

http://dx.doi.org/10.1109/ISANP.2017.8228782

N.B. When citing this work, cite the original published paper. 


\title{
A Multilayer-Stacked UWB Yagi Antenna
}

\author{
Kang Wan, Chao Xie, Yan Zheng, Jungang Yin \\ College of Electrical and Information Engineering, \\ Hunan University \\ Changsha, Hunan, 410082, China \\ yinjungang126@126.com \\ Jian Yang \\ Department of Electrical Engineering, \\ Chalmers University of Technology \\ Gothenburg, S-412 96, Sweden
}

\begin{abstract}
By taking advantage of a wideband bow-tie antenna in the Yagi configuration, a novel multilayer-stacked ultra-wideband (UWB) Yagi antenna is proposed. First, the fundamental 3-layered UWB Yagi model is studied with respect to a few critical dimensions. Then, several directors are added to form a multilayer-stacked Yagi antenna, where the width of the directors is optimized in a monotonic decreasing manner. A sixlayered UWB Yagi antenna is studied by simulations. It covers 3.4-10.6 GHz with its return loss better than $10 \mathrm{~dB}$, and it achieves a high directivity up to $13 \mathrm{dBi}$ and a relative cross-polar level better than $-10 \mathrm{~dB}$ in the whole band.
\end{abstract}

\section{Keywords-Multlayer-stacked; UWB; Yagi-Uda antenna}

\section{INTRODUCTION}

FCC in 2002 declared the ultra-wide band (UWB) ranging from $3.1 \mathrm{GHz}$ to $10.6 \mathrm{GHz}$ as a license free band. During recent decades, the UWB antennas have found many applications in radar, telecommunications, wireless sensor networks, remote sensing and so on [1]-[4]. In recent years, Yagi and quasi-Yagi antennas have drawn much attention because of high gain and simple structure [5]-[6]. In our previous research on feeds for reflector antennas, we presented the gain enhancement and matching improvement of a bow-tie antenna with a stacked parasitic patch on top [7]. The present study extends the use of the parasitic patches and investigates the effect of various parameters on antenna performance.

\section{FUNDAMENTAL 3-LAYERED UWB YAGI MODEL}

First, a planar bow-tie dipole is investigated as a driver of the Yagi antenna, whose petal is constructed by a rectangular and a semicircular patch, whereas the central of the dipole is cut into straight edges for connecting an UWB balun to a ground plane. The surface currents of the driver dipole at 3 and $11 \mathrm{GHz}$ are simulated by HFSS, as shown in Fig. 1. It can be observed that most of the currents is distributed along the edges near the feed gap at both frequencies. Therefore, a number of closely-spaced identical parallel patch bars can be used as directors, as shown in Fig. 2(a). If we increase the width of the bars and reduce the spacing until they join together, then the patch bars will be evolved into a solid patch, as depicted in Fig. 2(b). The S-parameter and co- and cross- polar far-field components of the patch bars and the solid patch are compared by using CST MWS. The results exhibited in Fig. 2(c) and (d) indicate that the two director configurations lead to very similar performance in both matching and radiation. For convenience, we use the solid patch for the director design.

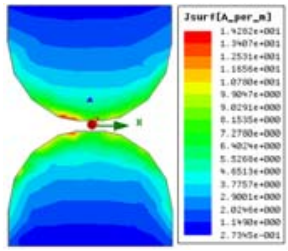

(a)

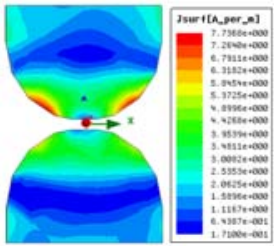

(b)
Fig. 1. Surface current on driver dipole: (a) $3 \mathrm{GHz}$, (b) $11 \mathrm{GHz}$.

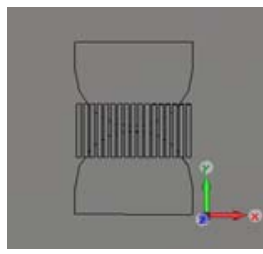

(a)

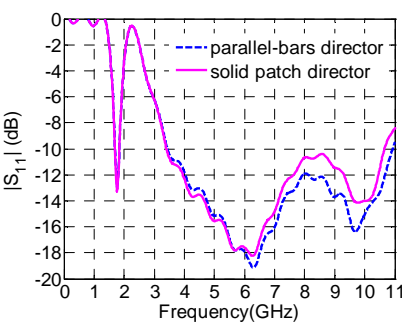

(c)

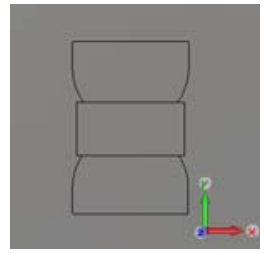

(b)

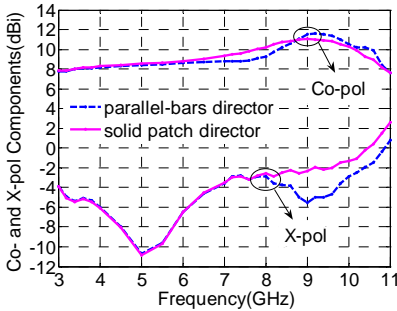

(d)
Fig. 2. Director evolution: (a) parallel-bars director, (b) solid patch director, (c) S-parameters, (d) co- and cross-polar directivities of the two configurations

The fundamental 3-layer-stacked Yagi antenna model is drawn in Fig. 3. The antenna is with air-substrate and consists of a planar bow-tie dipole as the driver, a patch as the director and the ground plane as the reflector, which are all made of copper plates with a uniform thickness $0.5 \mathrm{~mm}$. The driver dipole is fed by an UWB balun in connection with a coaxial 
cable that passes through the ground plane from beneath. Detailed dimensions are given in Table I.

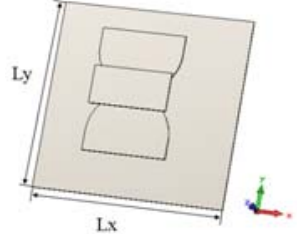

(a)

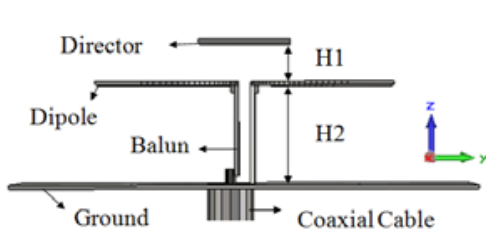

(c)

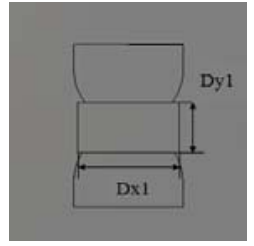

(b)

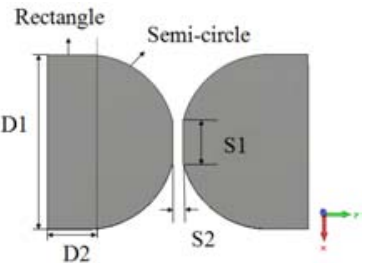

(d)
Fig. 3. Configuration of the 3-layered UWB Yagi antenna model: (a) 3D view, (b) top view, (c) side view, (d) source dipole.

TABLE I. PARAMETERS OF 3-LAYERED ANTENNA MODEL (mm)

\begin{tabular}{|c|c|c|c|c|c|c|c|c|c|}
\hline Parameter & Lx & Ly & Dx1 & Dy1 & H1 & H2 & D1 & S1 & S2 \\
\hline Value & 60 & 60 & 24 & 12 & 5 & 13 & 26 & 4.4 & 1.6 \\
\hline
\end{tabular}

\section{6-LAYER-STACKED UWB YAGI MODEL}

Based on the 3-layer-stacked model, we simply add more identical directors with same spacing H1 (see Fig. 3(c)) above the first director to form a multilayer-stacked UWB Yagi antenna. The relationship between its maximum gain in the operating ultra-wideband and the number of directors is investigated and compared with the linear narrow-banded Yagi antenna [8], as shown in Fig. 4(a). It can be found that the two Yagi antennas have a very similar trend for the gain curves, which indicate a saturation on the gains when the number of directors hits a certain value, 8, in this case; meanwhile, the gain of the UWB Yagi model is greater than its counterpart when the director number of the two is same.
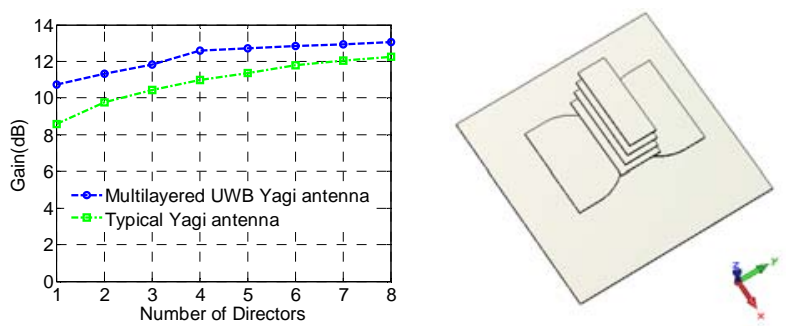

Fig. 4. (a) The gain versus the number of directors (the green curve is by courtesy of [8]), (b) A 3D view of a Yagi model with 4 directors.

Shown in Fig. 4(b) is a 6-layer-stacked model, for compromise on the gain and the compactness. However, the $\mathrm{S}_{11}$ parameters as well as the directivities of the 6-layered model with identical directors are not so good in the upper band (see Fig. 5). In order to improve the performance, the yaxis width of the 4 directors is optimized in a monotonic decreasing manner, while the x-axis length and the z-axis spacing are kept same as previous. Fig. 5 clearly shows that the model with decreased-width directors performs as well as the one with equal-width directors in the lower band, while the former greatly outperforms the latter in the upper band; the $S_{11}$ parameter is better than $-10 \mathrm{~dB}$ over $3.4-10.6 \mathrm{GHz}$, the directivities vary around $8-13 \mathrm{dBi}$ and the relative cross-polar level is below $-10 \mathrm{~dB}$ in the whole band.

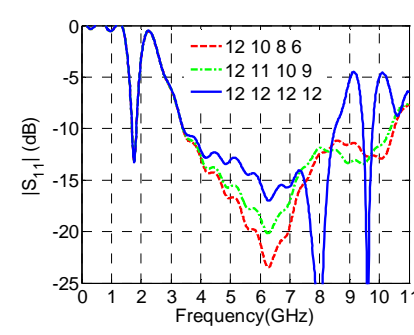

(a)

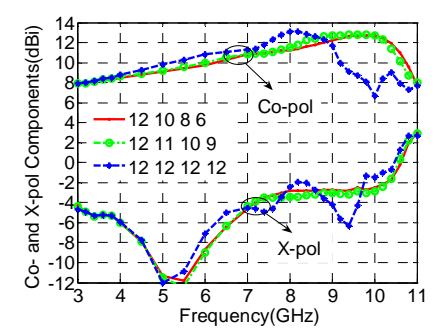

(b)
Fig. 5. (a) $\mathrm{S}_{11}$ and (b) co-polar directivity and cross-polar directivity vs. different y-axis width directors. The marking of 121086 means the width of the $1^{\text {st }}$ (closest to the driver dipole), $2^{\text {nd }}, 3^{\text {rd }}$ and $4^{\text {th }}$ director, respectively.

\section{CONCLUSION}

A six-layer-stacked UWB Yagi antenna has been proposed and optimized. The antenna can operate over $3.4-10.6 \mathrm{GHz}$ with return loss better than $10 \mathrm{~dB}$, the directivity between 8-13 $\mathrm{dBi}$, and the relative cross-polar level below $-10 \mathrm{~dB}$. Compared with the linear narrow-banded Yagi antenna with the same gain, this UWB Yagi antenna is more compact by using fewer directors, a candidate for UWB communication applications.

\section{ACKNOWLEDGMENT}

Jian Yang was supported by the funding from the European Union's Horizon 2020 research and innovation programme under grant agreement No 734798.

\section{REFERENCES}

[1] D. Awan, S. Bashir and N. Riauka, "Parametric study of UWB antenna loaded with stacked parasitic patch and reflector," 2013 Loughborough Antennas \& Propagation Conference (LAPC), Loughborough, 2013, pp. 358-362.

[2] Hussain, Jian Yang, Per-Simon Kildal, "Wideband Compact 4-Port Dual Polarized Self-Grounded Bowtie Antenna”, IEEE Trans. on Antennas Propagat., vol. 62, no. 9, pp. 4468-4473, Sept. 2014.

[3] Ali Al-Rawi, Ahmed Hussain, Jian Yang, Magnus Franzen, Charlie Orlenius, and Ahmed A. Kishk, "A new compact wideband MIMO antenna - the double-sided tapered self-grounded monopole array”, IEEE Trans. on Antennas Propagat., vol. 62, no. 6, pp. 3365-3369, 2014.

[4] J. Yang and A. Kishk, “The self-grounded Bow-Tie antenna”, 2011 IEEE international Symp. on Antennas Propag., Spokane, Washington, USA, July 3-8, 2011.

[5] W. Zhou, J. Liu and Y. Long, "A Broadband and High-Gain Planar Complementary Yagi Array Antenna With Circular Polarization," in IEEE Transactions on Antennas and Propagation, vol. 65, no. 3, pp. 1446-1451, March 2017.

[6] O. Kramer, T. Djerafi and K. Wu, "Vertically Multilayer-Stacked Yagi Antenna With Single and Dual Polarizations," in IEEE Transactions on Antennas and Propagation, vol. 58, no. 4, pp. 1022-1030, April 2010.

[7] C. Xie, J. Yin and et al., "An Ultra-Wideband Dipole with a Director as a Feed for Reflector Antennas". IEEE Antennas and Wireless Propagation Letters, DOI: 10.1109/LAWP.2016.2634002, 2017.

[8] W. L. Stutzman and G. A. Thiele, Antenna Theory and Design, 2nd ed. New York: Wiley, 1998. 